\title{
Corpus
}

4 | 2005

Les corpus politiques : objet, méthode et contenu

\section{Vers une logométrie intégrative des corpus politiques médiatisés. L'exemple de la subjectivité dans les débats-panel britanniques}

Laurent Rouveyrol

\section{CpenEdition}

\section{Journals}

Édition électronique

URL : http://journals.openedition.org/corpus/293

DOI : $10.4000 /$ corpus.293

ISSN : 1765-3126

Éditeur

Bases; corpus et langage - UMR 6039

Édition imprimée

Date de publication : 1 décembre 2005

ISSN : 1638-9808

Référence électronique

Laurent Rouveyrol, « Vers une logométrie intégrative des corpus politiques médiatisés. L'exemple de la subjectivité dans les débats-panel britanniques », Corpus [En ligne], 4 | 2005, mis en ligne le 01 septembre 2006, consulté le 08 septembre 2020. URL : http://journals.openedition.org/corpus/293 DOI : https://doi.org/10.4000/corpus.293

Ce document a été généré automatiquement le 8 septembre 2020

(C) Tous droits réservés 


\title{
Vers une logométrie intégrative des corpus politiques médiatisés. L'exemple de la subjectivité dans les débats-panel britanniques
}

\author{
Laurent Rouveyrol
}

Introduction

1 Suivant un modus vivendi bien établi, les analyses de corpus se rangent assez distinctement en deux catégories dominantes que sont les approches qualitatives d'une part et quantitatives d'autre part. Il est consensuellement admis que le recours à l'une ou l'autre de ces pratiques est souvent déterminé par la taille du corpus à explorer. Les chercheurs se situant dans une perspective quantitative reprochent à ceux de l'autre camp de se fonder sur des intuitions, d'aboutir à une herméneutique trop subjective sans tenir compte de la relativité des phénomènes au sein d'un corpus donné; les «qualitatifs» sont souvent convaincus qu'une analyse quantitative oblitère les spécificités génériques d'un corpus et ne dépasse pas la stricte matérialité textuelle, la réalité formelle. Pour être plus clair, les uns « font de l'explication de texte», les autres « comptent les mots».

2 Il conviendrait de replacer les différents axes au sein d'une perspective épistémologique plus globale en prenant en compte les modes liées aux différentes époques: le positivisme strict, le scientisme d'abord appliqué aux sciences humaines pour les constituer en tant que telles - c'était sans doute là une étape indispensable s'est progressivement adapté vers une phénoménologie plus ou moins assumée, permettant de considérer les sujets dans leur environnement socioculturel.

3 La dichotomie qualitatif / quantitatif renvoie à des problématiques beaucoup plus larges, qui ne font rien d'autre qu'interroger la notion de scientificité. Les approches qualitatives qui se donnent pour objet de rendre compte de fonctionnements langagiers et tentent de mettre au jour des stratégies au moyen de concepts pointus dépassent le niveau de l'intuition et d'une herméneutique aporétique'. De même, certaines 
approches quantitatives, en s'intéressant à des rapports de collocations, en permettant un étiquetage qualitatif des corpus (par des logiciels mis en place en amont tels que Treetagger pour l'anglais et Cordial pour le français ${ }^{2}$ ) font bien plus que simplement compter les mots : la nature grammaticale des segments est matérialisée, ce qui est un pas décisif vers un traitement qualitatif.

Nous voudrions suggérer d'une part que la réalité n'est plus aussi tranchée que la représentation parfois suggérée et d'autre part qu'il existe des moyens de concilier les deux aspects en aucune façon incompatibles; nous les considérons complémentaires. [Pourtois et Desmet 1997 : 31] remarquent d'ailleurs que : « le débat entre l'orientation quantitative et l'orientation qualitative est ancien. Pourtant, à l'heure actuelle, des tentatives de rapprochement se manifestent ».

1. Vers un modèle intégratif

5 La démarche que nous voudrions exposer ici consiste principalement à placer les spécificités génériques du corpus au centre d'un modèle d'analyse mis au point pour rendre compte de la variation des sujets dans un cadre situationnel donné. Nous proposons de prendre pour base la parole de deux politiciens britanniques lors d'un débat-panel (l'émission Question Time). Nous tenterons de rendre compte de l'éthos discursif, tel qu'il se détermine par l'analyse de stratégies et de modes d'implication du locuteur dans le discours. Il s'agit d'apporter des réponses à deux questions, posées par [Pourtois et Desmet $1997: 24]$ :

[...] Comment appréhender scientifiquement la signification subjective? Et comment saisir par un système de connaissances objectives les structures de signification subjectives?

1.1 Le corpus: Question Time

6 Notre corpus est constitué d'une émission de télévision nommée Question Time, produite par la B.B.C depuis 1979. Le jeudi soir, à 22h.30, elle attire plus de huit millions de téléspectateurs devant leur poste de télévision. Des politiciens et personnalités civiles en vue sont invités à faire partie d'un panel pour répondre aux questions que posera le public sur les thèmes d'actualité de la semaine. Un journaliste régule la parole et conduit l'émission à la manière d'un chef d'orchestre, permettant aux uns de parler et imposant aux autres de se taire.

7 Cette émission constitue donc une véritable « interaction » à plusieurs niveaux. Il s'agit d'une interaction verbale au cours de laquelle interviennent les participants faisant partie du plateau, c'est le niveau interne. ${ }^{3}$. Il existe un niveau externe, qui est la raison d'être de l'émission: tout programme télévisuel est censé mettre en place une interaction entre le téléspectateur et le plateau. Enfin, si l'on garde à l'esprit que Question Time est une émission politique, comme son nom le suggère ${ }^{4}$, il existe une autre interaction au sens strict, qui naît de la co-présence des politiciens et des électeurs(télé)spectateurs. Cette dernière interaction est irrémé-diablement liée à la vie politique contemporaine et porte des orientations perlocutoires très précises: l'élection de tel ou tel. Cette perspective correspond à la notion d'interaction, développée par [Vion $1992: 17]$ :

[...] toute action conjointe, conflictuelle ou coopérative, mettant en présence deux ou plus de deux acteurs. À ce titre, il recouvre aussi bien les échanges conversationnels que les transactions financières, les jeux amoureux que les matchs de boxe. 
Nous retiendrons pour cette étude une émission présentée par Peter Sissons ${ }^{5}$, ayant eu lieu en mars 1993, après la quatrième défaite consécutive des Travaillistes aux Elections Générales. Le panel était composé ce soir là de Kenneth Baker (député conservateur), de Pauline Green (député européen travailliste), de David Starkey (historien) et de Moira Constable (haut-fonctonnaire en charge du « Rural Housing Trust »). Les deux locuteurs que nous allons étudier plus précisément seront les politiciens, Baker et Green. Mais avant de présenter le modèle d'analyse, il convient de s'interroger sur la nature des éléments qui contribuent à réaliser une implication subjective ou intersubjective dans une telle situation de discours.

\subsection{Question Time et l'implication subjective}

$9 \quad$ La question de la subjectivité / intersubjectivité dans le discours est des plus épineuses et a passionné les linguistes depuis la nuit des temps. Elle ne sera pas réglée ici, autant le dire tout de suite; ce que nous souhaiterions, c'est tenter de proposer une problématisation de l'implication subjective, en fonction du genre étudié.

Les membres du public, ainsi que les invités formant le panel sont là pour donner leurs opinions à propos des questions posées. Chaque énoncé renvoie donc à une forme de subjectivité, quelle que soit sa forme. Cette constatation qui n'a l'air de rien, ne fait rien d'autre qu'exclure les représentations linguistiques du langage selon lesquelles il y aurait d'une part des énoncés neutres, et d'autre part des énoncés modaux, inscrivant une forme de subjectivité. La distinction scholastique modus/dictum ne peut être opérante ici qu'à partir du moment où l'on est assez large d'esprit pour considérer que le dictum est lui aussi la trace du travail subjectif d'un sujet parlant, même si ce travail de sélection des termes et des structures ainsi que d'autres activités langagières, n'encode pas directement un jugement de valeur assumé comme tel. Cela ne veut pas dire que tous les termes sont au même niveau et encodent la même subjectivité. Nous sommes d'avis qu'il existe des degrés d'implication divers et que le degré 0 ne fait pas partie de l'activité langagière, car un énoncé est toujours le produit du travail conscient ou inconscient d'un sujet parlant et pensant, faisant partie d'un environnement socioculturel donné.

11 Nous rejoignons alors la vision de [Fairclough 1995: 36] concernant la notion de modalité :

The concept of 'modality' is used in a very general way to cover features of texts which express speakers' and writers' attitudes towards themselves, towards their interlocutors and towards their subject-matter' (Fowler et alii, 1979: 200). Choices of pronouns, modal auxiliaries, speech acts, and many others, are included within modality.

Le discours est nécessairement soumis à l'activité des sujets; cette activité est consciente ou inconsciente et le linguiste la constate a posteriori, lui donnant éventuellement le nom de stratégie. Dépasser la dichotomie présence / absence de subjectivité ou encore subjectif / objectif est une nécessité qu'impose notre corpus, il convient donc de s'interroger sur la nature de ces «many others » qui font partie de la notion de modalité et que le cadre situationnel induit. Fairclough mentionne les actes de langage, cela nous oriente vers une conception plus large de l'activité des sujets que le simple fait de choisir des mots. L'attitude pragmatique des uns face aux autres, le comportement interactionnel des locuteurs, le choix des activités cognitivo-discursives (narration, description, argumentation) sont des éléments à prendre en compte dans un cadre comme le nôtre. 


\subsection{Le modèle d'analyse}

Dans sa conception, le modèle intègre les réflexions précédentes et a pour ambition de s'appuyer sur la matérialité du texte tout en permettant de la dépasser en considérant le niveau des activités co-construites.

\subsubsection{Les sources conceptuelles}

14 Le modèle tire sa source de trois cadres théoriques compatibles et complémentaires. L'aspect interactionnel, énonciatif ainsi que le côté générique et discursif complètent une série de marqueurs explicites. On part donc des locuteurs pour descendre jusqu'au niveau microlinguistique.

15 L'aspect interactionnel renvoie à la théorie des interactions verbales développée par Vion depuis 1992. On retiendra notamment le « modèle à cinq places ", qui a vu le jour dans «la gestion pluridimensionnelle du dialogue " [Vion 1995] et les mises en scène énonciatives [Vion 1998-b]'. Ces concepts constituent l'ossature essentielle de notre modèle. L'espace interactif que les interactants construisent et modifient à loisir prend appui sur cinq rapports de place. La place institutionnelle concerne le cadre générique a priori, le rapport peut être symétrique ou asymétrique : ami / ami, professeur / élève. La place modulaire renvoie aux sous-genres développés localement par les coparticipants : module conversationnel, polémique. La place subjective renvoie à l'image que se donnent individuellement et interactivement les communicants: rapport expert / non expert. La place discursive renvoie aux tâches cognitivo-discursives que les sujets entreprennent : choisissent-ils une narration, une description? Font-ils plutôt le choix d'argumenter? Enfin, la place énonciative, la plus fine, permet de descendre profondément dans la structure microlinguistique de l'énoncé. C'est à ce niveau que la notion de mise en scène énonciative est pertinente. On se demande comment le locuteur organise les voix qui traversent son discours.

La «Critical Discourse Analysis» mise au point par [Fairclough 1995, 1998] offre une théorisation des genres et des discours en accord avec l'aspect médiatisé de l'interaction étudiée ici. Quelle relation le langage entretient-il avec les processus sociaux ? Telle est la question posée. Fairclough propose une vision intégrative de la modalité que nous avons déjà mentionnée. Le fait de vouloir identifier des « ordres de discours» (inspirés de Foucault) dans les productions des locuteurs autorise une distinction très heuristique entre genre (communicationnel) et discours (intégrant la notion de point de vue). Ainsi l'ordre de discours d'un domaine social / institutionnel donné est constitué de tous les types discursifs le traversant. L'approche rend donc compte d'un dynamisme discursif et d'une hétérogénéité compositionnelle.

Enfin, les travaux de [Biber et al. 1999] concernant la définition des marques de «stance ${ }^{7}$ » en anglais ont été intégrés. Cette approche taxinomiste réalisée à grande échelle permet au besoin d'établir des comparaisons statistiques avec d'autres « registres de discours » tels que le roman, la conversation, le discours de la presse écrite, ou bien le discours académique.

1.3.2 Informatisation du modèle

18 Le modèle a été conçu pour être intégré à l'environnement informatique du logiciel Systemic Coder, créé par M. 0'Donnell' ${ }^{8}$. Ce logiciel, qui est un étiqueteur manuel, permet d'attribuer des traits, des propriétés, à des segments de corpus. Une fois le codage terminé, le linguiste se trouve face à une base de données, interrogeable à loisir et peut fonder ses raisonnements à partir de statistiques plus ou moins poussées : il peut s'agir 
de simples pourcentages ou bien de T-stats, appliquant la loi de Student-Fisher à partir d'un échantillonnage donné.

L'avantage d'utiliser un logiciel comme point de départ d'une analyse prenant pour objet le discours politique réside dans le fait que le linguiste n'est pas la proie de sa propre subjectivité mais considère comme pertinent ce que les statistiques imposent à son regard. Le modèle s'organise en quatre volets ${ }^{10}$.

-a) Les paramètres pragmatiques (type de locuteur, parti, profession, sexe).

-b) Les paramètres génériques et discursifs ${ }^{11}$ : les genres de discours renvoient à la plaisanterie, la polémique, l'interview, le débat ; les types de discours concernent l'ordre politique, ordinaire, médiatique et la propagande ${ }^{12}$; les types de séquences ${ }^{13}$ sont liés à l'argumentation, la description, le récit, l'explication.

-c) Des positionnements énonciatifs : nous nous intéressons ici aux mises en scène.

-d) L'utilisation de termes exprimant une «stance » directe (épistémique, attitudinelle et méta-linguistique), suivant les catégories de [Biber et al. 1999] : la stance lexicale comprend les termes axiologiques (beau, laid, horrible); la stance grammaticale met en perspective des unités telles que les auxiliaires modaux, les introducteurs de complétives de type I think, les adverbes de degré.

L'ensemble de la grille regroupe en plusieurs systèmes, parfois indépendants, quelques 140 critères. L'avantage de mettre en place des systèmes indépendants réside dans le fait de pouvoir les coder en simultané sur un même segment, car seuls sont exclusifs les traits faisant partie d'un même système. Naturellement, le logiciel n'accepte pas deux fois le même trait dans deux systèmes différents. Il n'y a rien d'étonnant à cela puisque le but consiste à établir une taxinomie. Si nous prenons les systèmes conçus pour informatiser les mises en scène énonciatives, nous aboutissons au schéma suivant : [graph manquant]

On doit noter tout d'abord que le concept de «mise en scène » «ne présuppose pas un sujet parlant conscient et autonome mais s'inscrit dans une approche interlocutive de l'énonciation ». Pour [Vion 1998-b] :

La notion de mise en scène concerne la façon dont les sujets construisent les opinions qu'ils mettent en circulation. Il s'agit, notamment, de savoir quelles sont les sources énonciatives construites dans les messages et de répondre à des questions comme: 'Le locuteur donne-t-il l'impression de parler seul ?', 'Fait-il parler d'autres personnes?', 'Ces autres voix sont-elles identifiables ou non?', 'Comment ces diverses voix s'articulent-elles dans la parole du locuteur?' et 'Comment celui-ci se positionne-t-il vis-à-vis des opinions ainsi construites ?'

Les cinq modes que le schéma présente en cinq systèmes indépendants peuvent se relier en fonction des différentes situations que les co-participants initient. Des mariages contre-nature sont théoriquement possibles mais à proscrire: il serait contradictoire de coder d'un côté un segment comme appartenant à l'unicité explicite (le mode du je pense que) et d'un autre le trait énonciation historique dans le mode de l'effacement. En revanche, il paraît très intéressant de montrer qu'un énoncé relève à la fois d'un acte indirect et d'une opposition exophonique, comme dans : "but not whilst the IRA are killing and maiming ". Par cet énoncé, Pauline Green répond à la question de savoir s'il faut négocier avec l'IRA. Elle exprime d'une part son opposition vis-à-vis du groupe mentionné absent (opposition exophonique) et d'une certaine façon, en même temps, elle les prévient (acte indirect) qu'elle ne veut pas négocier, tant que les massacres ne cessent pas. 
De même, nous nous sommes aperçu après le codage des énoncés prononcés par les politiciens, que nombreux étaient les segments partageant à la fois un trait du mode parallèle (l'énoncé va dans le même sens que celui d'un autre locuteur) et du mode de l'opposition (l'énoncé s'inscrit contre un autre énoncé). Cela peut paraître contradictoire et pourtant le discours politique n'est rien d'autre que le fait de parler avec l'appui de certains contre d'autres. Ainsi dans le cas de l'IRA toujours, Green dira : "you are not going to win by this sort of activity », en prêtant sa voix à Gordon Wilson, qui devait rencontrer les membres de l'IRA. C'est donc plutôt dans une combinaison de traits que la cohérence est à reconstruire.

2. Les politiciens sous les feux de la rampe

Le temps est venu de nous intéresser au discours de deux politiciens qui sont au premier plan dans une émission diffusée en 1993. Un politicien est par nature une instance locutive complexe; il parle en tant que représentant de son parti, en tant que représentant d'une frange électorale, en tant que membre du gouvernement, de l'opposition etc. Loin de faciliter l'analyse, cette fragmentation pragmatique ne la rendra que plus riche.

Les politiciens sont un peu la raison d'être du débat et font le lien entre les médias, le public et l'institution. Nous allons focaliser notre attention sur le discours de Kenneth Baker (ancien ministre, conservateur) et de Pauline Green (député travailliste, Labour), au cours de deux thèmes débattus : l'IRA et la Russie.

Notre façon de procéder sera de sélectionner les domaines à fouiller à partir de la grille et des T-stats. Lorsque l'écart type révèle que nous sommes en présence d'une divergence significative, un calcul se met en place et en fonction du nombre de segments concernés, établit une prédiction de reproductibilité des données. Ainsi lorsque le signe +++ apparaît à côté d'un trait, cela signifie que l'écart entre les deux locuteurs se reproduirait à $98 \%$, quel que soit le nombre de segments s'ajoutant à l'échantillon. Nous avons codé en tout 237 segments pour les deux locuteurs, ce qui représente au moins 33180 décisions pour les 140 traits que le modèle fédère.

2.1 Les politiciens et les thèmes

Le choix des thèmes de l'IRA et de la Russie a été déterminé par le fait que la production des deux locuteurs était proportionnellement déséquilibrée. C'est Baker qui domine pour l'IRA, mais Green parle beaucoup plus de Russie que lui.

\begin{tabular}{|l|l|l|l|l|l|l|l|l|l|l|}
\hline & Pauline & GREEN & & & & Kenneth & BAKER & \\
\hline \hline TRAIT & $\%$ & $\mathrm{~N}$ & $\mathrm{~T}$ & & $\%$ & $\mathrm{~N}$ & $\mathrm{~T}$ & \\
\hline \hline Interaction & $100 \%$ & 107 & 0.00 & & $100 \%$ & 130 & 0.00 & \\
\hline \hline Thèmes & & 107 & & & & 130 & & \\
\hline \hline Russie & $40.2 \%$ & 43 & 3.79 & +++ & $18.5 \%$ & 24 & 3.79 & +++ \\
\hline \hline IRA & $59.8 \%$ & 64 & 3.79 & +++ & $81.5 \%$ & 106 & 3.79 & +++ \\
\hline
\end{tabular}


$60 \%$ IRA, $40 \%$ Russie. Il n'en va pas de même pour Baker: $82 \%$ de son discours correspond à l'IRA et seulement $18 \%$ traite de la Russie. On remarque d'ailleurs que les $T$-stats avec trois croix nous incitent à rechercher des réponses de ce côté.

2.2 Analyse comparative

31 A la lecture globale du tableau ${ }^{14}$, on est frappé par la similarité des stratégies des deux locuteurs, pourtant de tendances idéologiques radicalement antagonistes; les points de divergence significatifs sont finalement assez réduits. Rappelons que Baker est conservateur et a eu toutes sortes de portefeuilles, cependant que Green est travailliste, dans l'opposition. Ils ont un profil différent, mais ils font sensiblement la même chose : ils tentent de séduire le spectateur pour le faire adhérer à leurs vues. Les différences significatives de stratégie portent sur les traits suivants :

- types de discours (vie quotidienne),

- appellatifs génériques (pronoms),

- unicité (implicite),

- dualité (actes indirects),

- parallélisme (porte-parole), représente (peuple),

- modaux et semi-modaux (semi-modaux),

- type de modalité (intrinsèque), epistemic type (certainty/doubt).

La totalité des divergences les plus statistiquement significatives tient en 9 systèmes et 9 traits. C'est très peu si l'on a à l'esprit que la grille contient 31 systèmes d'analyse qui totalisent environ 140 traits. Notre grille a pu paraître relativement complexe lors de son exposition, mais il est maintenant indéniable que la pluri-dimensionnalité la plus large est indispensable pour prendre en compte les variations parfois très fines des locuteurs. Les 9 systèmes se répartissent sur toute le modèle et touchent à toutes les dimensions de l'activité langagière : les choix de genre de discours, la construction de l'identité des co-participants, les voix convoquées, les positionnements énonciatifs et autres modulations, les modalisations microlinguistiques qu'intègrent les segments.

Le fait que les deux locuteurs partagent les mêmes stratégies sur plus de 120 autres critères nous oriente aussi vers l'idée qu'ils forment une communauté discursive cohérente, caractérisable de façon interne. Pour l'heure, voyons quel locuteur est responsable de l'écart en fonction des traits mentionnés plus haut; gardons à l'esprit que nous caractérisons pour l'instant un discours particulier en fonction de ce que l'autre ne fait pas. Nous allons nous concentrer plus spécifiquement sur les types de discours, le parallélisme et le type de modalité.

2.2.1 Types de discours, la vie quotidienne

Le trait singularisé est celui du discours de la vie quotidienne. Il oppose le discours de Green (34\%) à celui de Baker (15\%). C'est donc le discours de Pauline Green qui est ici responsable de l'écart, car c'est elle qui s'écarte le plus de la norme moyenne calculée à partir des deux locuteurs.

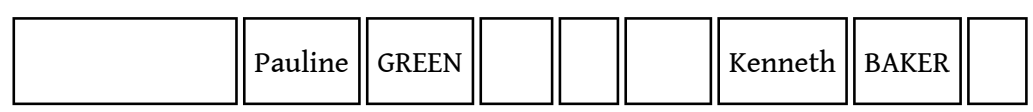

Corpus, 4 | 2005 


\begin{tabular}{|l|l|l|l|l|l||l|l||l|}
\hline TRAIT & $\%$ & $\mathrm{~N}$ & $\mathrm{~T}$ & & $\%$ & $\mathrm{~N}$ & $\mathrm{~T}$ \\
\hline \hline Types discours & $100 \%$ & 107 & 0.00 & & $100 \%$ & 130 & 0.00 & \\
\hline \hline Des médias & $1 \%$ & 1 & 1.89 & + & $5 \%$ & 7 & 1.89 & + \\
\hline \hline Politique & $51 \%$ & 57 & 1.55 & & $62 \%$ & 80 & 1.57 & \\
\hline \hline Vie quotidienne & $34 \%$ & 36 & 3.36 & +++ & $15 \%$ & 20 & 3.36 & ++ \\
\hline \hline Institutionnel & $4 \%$ & 4 & 1.07 & & $7 \%$ & 9 & 1.07 & \\
\hline \hline Propagande & $8 \%$ & 9 & 0.61 & & $11 \%$ & 14 & 0.61 & \\
\hline
\end{tabular}

Green : 36 segments, parmi lesquels :

105 that enough is enough

109 that what happened in Warrington was so abhorrent

110 we listened to the excuses of the IRA /

111 when they say: we don't want to hurt anybody / they say we don't want to hurt anybody / we didn't mean to kill children

Le marquage considéré pour identifier un tel discours est ici plutôt lexical, avec des expressions familières telles que a great deal of money ou des expressions (figées) telle que enough is enough. On peut aussi remarquer que, pour cette locutrice, le trait semble lié au fait de laisser parler les autres dans son propre discours.

Baker : 20 segments, parmi lesquels :

76 it's a very very honorable way actually

77 this man who has suffered himself so dreadfully to actually be going to speak in the Irish senate /

78 which is what he is going to do

79 and I think that

80 his voice will be listened to

On note que pour Baker, il existe une relation entre le marquage axiologique d'ordre principalement éthique et l'ordre de discours de la vie quotidienne. L'unicité semble aussi une caractéristique: implicite (honorable, dreadfully), générée par l'axiologisation du discours, mais aussi explicite, comme le montre le segment 79.

2.2.2 Le mode du parallélisme

Une fois encore, c'est le discours de Green qui nous fait considérer ce système.

\begin{tabular}{|l|l|l|l|l|l|l|l|l|l|}
\hline & Pauline & GREEN & & & & Kenneth & BAKER & \\
\hline \hline TRAIT & $\%$ & $\mathrm{~N}$ & $\mathrm{~T}$ & & $\%$ & $\mathrm{~N}$ & $\mathrm{~T}$ \\
\hline \hline Parallélisme & $100 \%$ & 107 & 0.00 & & $100 \%$ & 130 & 0.00 & \\
\hline \hline Non marqué & $59 \%$ & 63 & 1.53 & & $68 \%$ & 89 & 1.53 & \\
\hline
\end{tabular}




\begin{tabular}{|l|l|l|l|l|l|l|l|l|}
\hline diaphonique & $1 \%$ & 1 & 0.81 & & $2 \%$ & 3 & 0.81 \\
\hline \hline exophonique & $5 \%$ & 5 & 0.50 & & $6 \%$ & 8 & 0.50 & \\
\hline \hline polyphonique & $10 \%$ & 11 & 0.49 & & $12 \%$ & 16 & 0.49 & \\
\hline \hline porte-parole & $21 \%$ & 23 & 2.89 & +++ & $8 \%$ & 11 & 2.89 & +++ \\
\hline
\end{tabular}

\begin{tabular}{|l|l|l|l|l|l|l|l|l|l|}
\hline & Pauline & GREEN & & & & Kenneth & BAKER & \\
\hline \hline Représente & $100 \%$ & 107 & 0.00 & & $100 \%$ & 130 & 0.00 \\
\hline Institution & $9 \%$ & 10 & 0.45 & & $8 \%$ & 10 & 0.45 \\
\hline Peuple & $7 \%$ & 7 & 3.00 & +++ & $0 \%$ & 0 & 3.00 \\
\hline Autre & $6 \%$ & 6 & 2.20 & $1 \%$ & 1 \\
\hline
\end{tabular}

Cette locutrice se pose clairement en porte-parole du peuple. Si l'on cumule le parallélisme polyphonique et la mise en scène du porte-parole, on s'aperçoit que c'est Green qui emmène le plus de monde avec elle : $31 \%$, alors que Baker ne totalise que $21 \%$. Les segments de Green comprenant le trait porte-parole sont les suivants :

858 and part of what we've been doing to date / is actually putting money in aid 859 always tied to what we could get out of it in terms of benefit 860 and one of the things we need to do

41 Les énoncés où Baker se pose en porte-parole sont par exemple :

893 and we must do

894 everything we can at the Vancouver summit / through aid and through assistance Le pronom we ainsi que les auxiliaires modaux semblent jouer un rôle important dans ces énoncés. Les énoncés de Green porte-parole du peuple sont les suivants :

316 we need to understand that

332 and only by discussions and negotiations / will we get that political decision making On voit que, si le segment 316 ne pose pas de problème quant à l'identification du trait peuple, le segment 332 est plus douteux : le GN political decision making pourrait aussi nous pousser à coder le trait autre, qui correspond souvent à une combinaison des choix proposés dans le système.

Baker est clairement du côté de l'institution: il la représente à $91 \%$. Les énoncés partageant ce trait sont :

290 we have tried in the last three years under Peter Brook and now under Patrick Mayhew to get talks going again in Northern Ireland

Il se produit chez Green un phénomène très intéressant. On voit qu'elle est porteparole de l'institution à $43 \%$ et du peuple à $30 \%$. Green représente davantage l'institution que le peuple, mais sa capacité à se poser en porte-parole du peuple est beaucoup plus 
importante que celle de Baker: le trait de représentante du peuple caractérise son discours non pas de façon interne mais par rapport à celui de Baker.

En ce qui concerne l'institution, les segments sont pour Green :

859 always tied to what we could get out of it in terms of benefit

860 and one of the things we need to do

861 is make sure that our whole our whole system is looking at providing that

La représentation opérée par ces deux locuteurs est conforme à leur profil idéologique : Baker est conservateur et son parti est au gouvernement, il en est donc solidaire et donne sa voix à ses confrères, honorable friends. Green prête sa voix à ceux qui subissent l'institution, au peuple, qu'elle choisit de représenter en tant que député travailliste.

2.2.3 Le type de modalité

Le type de modalité est lié au système des modaux et semi-modaux ${ }^{15}$ : le fait d'utiliser davantage un auxiliaire tel que Need a des répercussions sur le type de modalité mise en discours.

\begin{tabular}{|c|c|c|c|c|c|c|c|c|}
\hline & Pauline & GREEN & & & & Kenneth & BAKER & \\
\hline TRAIT & $\%$ & $\mathrm{~N}$ & $\mathrm{~T}$ & & $\%$ & $\mathrm{~N}$ & $\mathrm{~T}$ & \\
\hline Type Modalité & $100 \%$ & 107 & 0.00 & & $100 \%$ & 130 & 0.00 & \\
\hline Intrinsèque & $18 \%$ & 19 & 3.08 & +++ & $5 \%$ & 7 & 3.08 & +++ \\
\hline Extrinsèque & $3 \%$ & 3 & 1.44 & & $7 \%$ & 9 & 1.44 & \\
\hline Non définissable & $3 \%$ & 3 & 1.93 & + & $0 \%$ & 0 & 1.93 & + \\
\hline Sous-Type Epistémique & $100 \%$ & 107 & 0.00 & & $100 \%$ & 130 & 0.00 & \\
\hline doute/certitude & $1 \%$ & 1 & 2.48 & +++ & $8 \%$ & 10 & 2.48 & +++ \\
\hline Réalité & $7 \%$ & 7 & 0.12 & & $7 \%$ & 9 & 0.12 & \\
\hline kn-perspective & $18 \%$ & 19 & 0.72 & & $22 \%$ & 28 & 0.72 & \\
\hline
\end{tabular}

Ce tableau montre que le type de modalité permet de caractériser les deux discours de façon positive et non plus par contraposition. On assiste à un véritable chassé-croisé :

\begin{tabular}{|l|l|}
\hline Baker & Green \\
\hline \hline [- intrinsèque $]$ & [+ intrinsèque $]$ \\
\hline \hline [+ doute/certitude $]$ & [- doute/certitude $]$ \\
\hline
\end{tabular}


51

Autrement dit, les deux systèmes sous-type épistemique et type de modalité se font écho dans les résultats et enregistrent deux fois une divergence de stratégie. On a envie de dire que c'est logique puisque les modalités intrinsèques sont exclusives des modalités épistémiques et que, par conséquent, ce qui n'est pas catégorisé sous une rubrique l'est forcément dans la rubrique complémentaire. Si une telle chose était vraie, alors ce serait le trait [+/- extrinsèque] qui particulariserait le discours de Baker. On s'aperçoit, en examinant les chiffres pour l'épistémique, que Baker a fait le choix de concentrer ses énoncés sur l'axe du doute et de la certitude (constituant $8 \%$ de son discours), cependant que Green a fait le choix d'utiliser une modalité radicale (constituant $18 \%$ de l'ensemble). On peut donc conclure que les énoncés de Baker, du fait de leur répartition inégale dans le système épistémique, ne sont pas par défaut épistémiques, mais procèdent d'un choix stratégique privilégiant l'axe doute/certitude.

Voici des segments concernés par le trait intrinsèque chez Green :

(138 I think as a British politician)

139 / we could not

140 and should not be talking to the IRA

Chez Baker :

893 and we must do

894 everything we can at the Vancouver summit / through aid and through

2.3 Vers une cohérence des stratégies2.3.1 Essai de synthèse à propos de l'analyse comparative

En reprenant les différents traits divergents et en les attribuant au locuteur qui réalise l'écart, on s'aperçoit que nous avons déjà matière pour proposer une analyse.

\begin{tabular}{|l|l|}
\hline Kenneth BAKER & Pauline GREEN \\
\hline \hline - Marques subjectives internes (appellatifs) & - Vie quotidienne \\
- Unicité implicite & - Actes indirects \\
- Modalité épistémique : & - Porte-parole du peuple \\
axe certainty/doubt & - Utilisation des semi-modaux : need \\
& - Modalité intrinsèque \\
\hline
\end{tabular}

Si l'on se contente de gloser ces traits pour chacun des locuteurs, on obtient le mouvement suivant:

- Green est résolument du côté du peuple qu'elle représente souvent, en choisissant surtout l'ordre de discours de la vie quotidienne. Elle n'hésite pas à être directe et se situe souvent dans le domaine radical de la modalité (intrinsèque chez Biber) ; en d'autres termes, son discours est orienté vers les modalités de l'action : ce qu'il faut faire, les actions à mener, ce dont nous avons besoin.

- Inversement, Baker modalise moins directement et joue sur le mode implicite. Son discours est plus politique. Il est en revanche plus directement impliqué dans la relation diaphonique interne et fait usage de pronoms matérialisant la présence des co-participants dans le débat. Il envisage les choses avec un recul intellectuel, en se situant sur l'axe très abstrait doute / certitude. C'est davantage en termes de raison qu'en termes d'action qu'il envisage les 
problèmes : Baker n'est pas vraiment intéressé par ce dont nous avons besoin ou ce qu'il faut

faire, mais plutôt par ce qui arrivera ou peut arriver.

2.3.2 Caractérisation interne des discours combinaisons obtenues :

1) Traits : débat argumentation politique unicité stance marquée : 2-propositions structures-complétives gouvernées-par-verbe knowledge-perspective, attribution explicite.

Nombre : 9

Exemple : I think they put themselves beyond the law and also beyond political debate / that is my view

2) Traits : débat description politique énonc-historic stance-non-marquée Nombre : 7

Exemple : And in internment / there is internment without trial

3) Traits : débat argumentation politique énonc-historic stance-non-marquée

Nombre : 6

Exemple : that what is different about this particular case - Warrington /

4) Traits : débat explication politique énonc-historic stance-non-marquée

Nombre : 4

Exemple : because they have had a political solution

Les choses sont claires. Sur quatre groupements opérés à partir des combinaisons les plus récurrentes, le trait argumentation sort deux fois, suivi par les traits description et explication. Le politicien est résolument dans le débat et son discours est politique. Les mises en scène révèlent que l'on passe d'un haut degré de subjectivité plus ou moins assumé (unicité implicite) à un effacement objectivant (énonciation historique); le mode de l'effacement, contre toute attente, s'impose sur 3 combinaisons. Voyons maintenant comment nos deux politiciens se situent par rapport à ce profil synthétique.

Les combinaisons pour Baker :

K.B1) Traits : débat argumentation politique unicité explicite 2-propositions structures-complétives gouvernées-par-verbe epistemic knowledge-perspective, attribution explicite

Nombre : 4

Exemple : I think they put themselves beyond the law and also beyond political debate / that is my view

K.B2) Traits : débat description politique énonc-historic stance-non-marquée

Nombre : 4

Exemple : And in internment / there is internment without trial 
K.B3) Traits : débat argumentation politique énonc-historic stance-non-marquée Nombre : 3

Exemple : that what is different about this particular case - Warrington /

K.B4) Traits : débat argumentation politique opolyphonique 2-propositions structurescomplétives gouvernées-par-verbe epistemic knowledge-perspective explicit

Nombre : 2

Exemple : and I don't think

Les trois premières combinaisons montrent que Baker correspond au politicien-type : il suit exactement le classement des combinaisons prototypiques 1,2 et 3 . Il s'écarte simplement du prototype en revenant à l'argumentation, là où l'explication apparaît dans l'ordre normatif en quatrième position. Autrement dit, le discours de Baker est parfaitement ce qu'un politicien doit produire. Cette adéquation pourrait être à nuancer parce que Baker a plus de segments que Green, ses stratégies personnelles pèsent donc plus sur la norme que tous les deux déterminent.

61 Les combinaisons de Green :

P.G1) Traits : débat argumentation politique unicité explicite. 2-propositions structures-complétives gouvernées-par-verbe sem-st-type epistemic knowledgeperspective

Nombre : 5

Exemple : I think

P.G2) Traits : débat explication politique énonc-historic stance-non-marquée

Nombre : 4

Exemple : because they have had a political solution

P.G3) Traits : débat description politique énonc-historic stance-non-marquée

Nombre : 3

Exemple : what Spain has done in the Basque country /

P.G4) Traits : débat argumentation politique énonc-historic stance-non-marquée

Nombre : 3

Exemple : and in so doing have marginalized the terrorists

P.G5) Traits : débat argumentation politique opolyphonique stance-non-marquée

Nombre : 3

Exemple : is try to support and encourage / democratic participative ha / government P.G6) Traits : débat description politique unicité explicite

2-propositions structures-complétives gouvernées-par-verbe knowledge-perspective, attribution explicite

Nombre : 2

Exemple : and I do believe that the Irish are intimately and inextricably involved in this

Un premier élément de commentaire est que, pour descendre jusqu'à deux occurrences, il faut attendre la sixième combinaison pour Green, ce qui montre que son discours est beaucoup plus mouvant, moins stéréotypé d'un point de vue général.

63 L'explication apparaît en deuxième position pour Green, alors que ce trait figure dans la quatrième combinaison prototypique. On peut dire que Green fait en quelque sorte moins souvent la même chose. Les quatre premières combinaisons sont assez équilibrées quantitativement : $5,4,3,3$ et 3 segments. 

est de rendre compte de la relativité des stratégies analysées. Dans l'approche que nous présentons, ces phénomènes ne sont pas uniquement matériels, car ils concernent des réalités aussi suprasegmentales que les positionnements énonciatifs. On s'aperçoit alors que la logométrie intégrative repousse certaines limites de la matérialité, si l'on se fonde sur la définition que donne [Mayaffre 2005] : « Avec la logométrie, l'analyste part toujours de faits linguistiques matériels massivement attestés (y compris dans leur absence ou leur rareté) pour commencer à réfléchir et le cas échéant inférer un positionnement idéologique ou une attitude politique. "Si l'on admet que les faits linguistiques matériels sont ceux que les statistiques mettent en lumière à partir de quelque phénomène que ce soit, alors nous sommes bien dans une orientation logométrique.

Enfin, nous espérons avoir montré qu'une quantification des données n'était pas incompatible avec une perspective interactionniste phénoménologique, replaçant le sujet parlant dans un environnement socioculturel, considérant les multiples recadrages et autres ruptures énonciatives comme faisant partie intégrante de l'activité de co-construction des discours. Nous espérons qu'une telle méthodologie contribuera à faire parler les textes et les discours de façon encore plus précise et heuristique. 


\section{BIBLIOGRAPHIE}

Adam J.-M. (1992). Les textes : types et prototypes. Paris : Nathan.

Biber D., Johansson S., Leech G., Conrad S. \& Finegan E. (1999). Grammar of Spoken and Written English. London : Longman.

Chomsky N. (2002). Media Control : The Spectacular Achievements of Propaganda. New York : Seven Stories Press.

Ducrot O. (1984). Le dire et le dit. Paris : Les Editions de Minuit.

Dujardin S. \& Rouveyrol L. (2004). « Emphase et variation individuelle dans le débat télévisé: paramètres acoustiques et pragmatico-énonciatifs ». Communication au congrès de l'ALOES, Association des Linguistes Oralistes de l'Enseignement Supérieur, Villetaneuse, avril 2004, à paraître dans les actes.

Fairclough N. (1995). Media Discourse. London : Arnold.

Fairclough N. (1998), « Political Discourse in the Media: An Analytical Framework », in A. Bell \& P. Garrett (éds), Approaches to Media Discourse. Oxford : Blackwell, pp. 142-162.

Fairclough N. (2001). « The Discourse of New Labour: Critical Discourse Analysis », in M. Wetherell, S. Taylor \& S. J. Yates (éds), Discourse as Data. London : Sage, pp. 229-266.

Mayaffre D. (2005). « Analyse du discours politique et Logométrie : point de vue pratique et théorique », Langage et Société 114 : 91-111.

O'Donnell M. (2002). Systemic Coder : <www.wagsoft. com/coder>.

Oakes M.P. (1998). Statistics for corpus linguists. Edinburgh : Edinburgh University Press.

Pourtois J-P. \& Desmet H. (1997). Epistémologie et instrumentation en sciences humaines. Liège : Mardaga.

Rouveyrol L. (2003). Etude pragmatique de la variation linguistique dans le débat politique médiatisé en anglais. Les modalités et marques d'implications du locuteur dans le discours. Thèse N.R, F. Dubois-Charlier (Dir), Aix-en-Provence.

Rouveyrol L., Maury-Rouan C. \& Vion R. (2005). « A linguistic toolbox for discourse analysis, towards a multidimensional handling of verbal interactions ", Discourse Studies 7, $3: 289-313$.

Scannell P. (éd) (1991). Broadcast Talk. London : Sage.

Viallon P. (1996). L'analyse du discours de la télévision. Paris : P.U.F.

Vion R. (1992). La communication verbale, Analyse des interactions. Paris : Hachette.

Vion R. (1995). « La gestion pluridimensionnelle du dialogue », Cahiers de linguistique française 17 : 179-203.

Vion R. (éd) (1998a). Les sujets et leurs discours. Enonciation et interaction. Aix-en-Provence :

Publications de L'Université de Provence.

Vion R. (1998b). «La mise en scène énonciative du discours », in B. Caron (éd), Proceedings of the 16th international Congress of Linguists, [CD-ROM]. Oxford : Elsevier Sciences. 


\section{NOTES}

1.. Voir [Vion 1998 a].

2.. Voir [Mayaffre 2005]

3.. La distinction entre plans internes et externes a été remarquée par tous les analystes du discours télévisuel (Scannell). Nous reprenons ici la terminologie de [Viallon 1996 : 38].

4.. Pour un anglophone, l'expression renvoie à la vie parlementaire et à l'épisode au cours duquel les députés peuvent poser des questions au gouvernement et au Premier Ministre à la Chambre des Communes.

5.. Ce journaliste est dorénavant remplacé par David Dimbleby.

6.. Pour un exposé complet de ces concepts en anglais et un exemple d'analyse, voir [Rouveyrol et al. 2005].

7.. Le terme est difficile à rendre en français, une glose acceptable serait :

«positionnement subjectif».

8.. Ce logiciel est mis gratuitement à la disposition des linguistes par son auteur : www.wagsoft.com/coder

9.. Pour [Oakes $1998: 10]$ : « The $t$ test tests the difference between two groups for normally-distributed interval data where the mean and standard deviation are appropriate measures of central tendancy and variability of the scores. We use a t test [...] whenever we are dealing with small samples (where either group has less than 30 items).»

10.. Pour des raisons d'espace, il n'est pas envisageable de détailler chaque catégorie et de donner une définition pour chaque trait. Nous renvoyons à notre thèse [Rouveyrol $2003: 145-198]$ pour de plus amples explications. Ce travail sera partiellement mis en ligne sur le site du laboratoire « Bases, Corpus, Langage », UMR 6039, Nice - Sophia Antipolis.

11.. Les catégories mentionnées ci-après ainsi que la distinction genre / type de discours, sont celles de la CDA.

12.. Après la lecture d'un opuscule de Chomsky (2002), le discours de «propagande » a été ajouté aux trois premiers, qui pour [Fairclough 1995] constituent l'ordre du discours du discours politique médiatisé. Pour [Chomsky 2002 : 26], en utilisant le discours de la propagande : "You want to create a slogan that nobody's going to be against, and everybody is going to be for. Nobody knows what it means, because it doesn't mean anything. Its crucial value is that it diverts your attention from a question that does mean something [...]. »

13.. Il s'agit là de la théorie séquentielle développée par [Adam 1992].

14.. Le logiciel génère un tableau de cinq pages qu'il n'est pas possible de reproduire dans son intégralité ici.

15.. Rappelons que nous avons adopté la catégorisation de [Biber et al. 1999], qui propose d'inclure dans les semi-modaux les expressions modales telles que have to ou be going to. 


\section{RÉSUMÉS}

Cet article présente une méthodologie spécifique destinée à traiter le discours politique médiatisé. Aussi bien qualitative que quantitative dans sa conception, la logométrie intégrative offre la possibilité de quantifier des stratégies de positionnements énonciatifs ou bien le recours à des activités cognitivo-discursives telles que la narration ou l'argumentation en même temps que des marqueurs textuels. Après le codage manuel d'un corpus échantillon, des statistiques sont générées par le logiciel Systemic Coder (O'Donnell, 2002) pour comparer la production discursive de deux politiciens d'opinions différentes ayant participé au débat panel Question Time en mars 1993. Les résultats montrent que seuls 9 traits sur 140 formant l'ensemble du modèle sont identifiés comme étant significativement divergents (Loi statistique de Student-Fisher). Les traits mis en exergue se répartissent sur l'ensemble de la grille, depuis les choix de genre et de mise en scène énonciative, jusqu'au recours à certains marqueurs modaux: cela montre la nécessité d'adopter une perspective pluridimensionnelle. Une caractérisation interne des discours comparés (analyse par groupement de cellules) révèle que la notion d'écart n'est pas suffisante pour reconstruire la cohérence des activités langagières au niveau d'un locuteur donné.

Towards an integrative logometry of mediatized political discourse. The example of subjectivity in British panel debates.

This paper presents a methodology designed to deal with mediatized political discourse. Integrative Logometry is aimed at bridging the gap between qualitative and quantitative perspectives, offering the possibility to quantify speakers' suprasegmental strategies like positioning processes or choices of activity types such as narration or argumentation as well as textual markers. After coding manually a sample corpus, statistics are set by O'Donnell's Systemic Coder (2002) to compare the discourse of two ideologically antagonistic politicians in the programme Question Time broadcast in March 1993. Results show that only 9 features out of 140 forming the entire model are identified as significantly divergent by the software generating $t$ stats. Yet the features highlighted span from discourse types and self positioning processes down to the choice of modal auxiliaries ; a multidimensional analysis is therefore indispensable. An internal characterisation of the two discourses compared (cell analysis) reveals that the notion of deviation is not sufficient to analyse the coherence of language activity in a given discourse.

INDEX

Mots-clés : débat-panel, logométrie intégrative, analyse de corpus, stratégies discursives, discours politique médiatisé, interaction verbale

\section{AUTEUR}

LAURENT ROUVEYROL

UNSA, BCL, Nice 